\title{
¿Puede un pulsómetro acortar la incapacidad temporal de los pacientes con cardiopatía isquémica?
}

\section{Can a heart rate monitor shorten the temporary disability of patients with ischemic heart disease?}

\section{Raúl Jesús Regal Ramos'}

1. Dirección Provincial del Instituto Nacional de la Seguridad Social de Madrid. España.

\section{Recibido: 14-12-15}

Aceptado: 26-12-15

\section{Correspondencia}

Raúl Jesús Regal Ramos.

Médico Inspector de La Dirección Provincial del Instituto Nacional de la Seguridad Social de Madrid. España

C/ López de Hoyos 169. Madrid. España.

Teléfono de contacto: 915907143

Correo Electrónico: raul-jesus.regal@inss.seg-social.es

ResUmen

Es habitual que los cardiólogos recomienden el uso de pulsómetros en determinados pacientes con cardiopatía isquémica que quieren retomar la práctica deportiva. Actúan como un elemento de seguridad que permite regular la intensidad del ejercicio. Al igual que el pulsómetro es una herramienta que ayuda a compatibilizar la cardiopatía isquémica con la práctica de determinados esfuerzos físicos deportivos ¿podría ser una herramienta que ayude a compatibilizar la cardiopatía isquémica con la realización de determinados esfuerzos físicos laborales? En aquellos casos en los que médicamente existe una recuperación suficiente para la reincorporación laboral pero el paciente mantiene dudas al respecto, el uso del pulsómetro podría proporcionarle esa seguridad que facilitase la reincorporación laboral.

Med Segur Trab (Internet) 2015; 61 (241) 480-485

Palabras claves: Incapacidad. Cardiopatía isquémica. Intensidad de ejercicio. Prescripción del ejercicio.

Abstract

Usually cardiologists recommend the use of heart rate monitors in selected patients with ischemic heart disease who want to resume a sport activity. It acts as a safety feature that regulates the intensity of exercise. In the same way that a heart rate monitor is a tool that helps to co-ordinate ischemic heart disease with carrying out certain exercises or physical efforts, could it be used as a tool to help reconcile ischemic heart disease for the completion of certain labor efforts? In certain cases in which there is sufficient medical recovery to return to work, but the patient keeps doubting, the use of the heart rate monitor would provide that security that would facilitate the return to work.

Med Segur Trab (Internet) 2015; 61 (241) 480-485

Key words: Disability. Coronary heart disease. Intensity exercise. Prescription of exercise. 


\section{INTRODUCCIÓN}

Practicando el ciclismo me he encontrado subiendo puertos de montaña a varios pacientes con cardiopatía isquémica que regulaban su esfuerzo por medio de pulsómetros. Esto me ha llevado a pensar si no podría ser el pulsómetro un instrumento que sirviese también para regular el esfuerzo que se realiza en una jornada laboral. Lógicamente, al igual que el cardiólogo solo permite en determinados casos de cardiopatía isquémica la realización de ciertos esfuerzos físicos, como el ciclismo, la utilización del pulsómetro en la reincorporación laboral solo podría ser aplicable en determinados tipos de cardiopatía isquémica y en determinadas profesiones.

La aptitud para la práctica deportiva, y de esfuerzos físicos en general, en pacientes con cardiopatía isquémica, viene dada por el riesgo de sufrir un nuevo episodio isquémico agudo. Partimos del hecho de que la respuesta aguda al ejercicio produce un aumento en las necesidades de $\mathrm{O}_{2}$ y de la actividad simpática que puede originar la aparición de espasmo coronario. Se produce un aumento del gasto cardíaco ${ }^{1}$, aumento del consumo de $\mathrm{O}_{2}$, incremento del retorno venoso, aumento de la contractilidad del miocardio y una la disminución de las resistencias periféricas (aunque el incremento del gasto cardíaco durante el ejercicio es siempre superior a la disminución de las resistencias periféricas, por lo que se produce una elevación de la presión arterial). Sin embargo un ejercicio físico aeróbico controlado a la larga mejora el control de los factores de riesgo, tiene efecto directo sobre la función endotelial, aumenta la capacidad funcional y disminuye el consumo de $\mathrm{O}_{2}$, lo que implica un menor trabajo cardíaco y una elevación del umbral de angina.

Cuando valoramos la capacidad laboral de cualquier paciente tenemos en cuenta las limitaciones derivadas de la enfermedad y los requerimientos de su trabajo. Para establecer los requerimientos de cada puesto de trabajo nos apoyamos en Guías de valoración profesional del Instituto Nacional de la Seguridad Social ${ }^{2}$ normativas específicas (RD 818/2009 que regula las aptitudes psicofísicas requeridas para obtener el permiso de conducción, Reglamento de la Unión Europea n. ${ }^{\circ} 1178 / 2011$ por el que se establecen requisitos técnicos relacionados con el personal de vuelo, etc.), informes de los servicios de prevención, etc. Para establecer las limitaciones derivadas de la cardiopatía isquémica nos apoyamos en los antecedentes del paciente, la clínica, la exploración física, las pruebas complementarias y los tratamientos. Entre las pruebas complementarias más utilizadas en la valoración de la cardiopatía isquémica están las pruebas de detección de isquemia (ergometría, eco de estrés o perfusión isotópica), pruebas de imagen (ECO, cardio-RMN...), el Holter y la coronariografía, entre otras.

El objetivo de este artículo es plantear si el pulsómetro, lo mismo que es una herramienta que hace compatible la cardiopatía isquémica con la práctica de determinados esfuerzos físicos deportivos, podría ser una herramienta que ayudase a compatibilizar la cardiopatía isquémica con la realización de determinados esfuerzos físicos laborales.

\section{¿EN QUÉ TIPO DE PACIENTES PODRÍA RECOMENDARSE EL USO DE PULSÓMETROS?}

La prescripción siempre sería individualizada en función del riesgo cardiovascular del paciente. Podemos estratificar el riesgo del paciente de sufrir un nuevo evento coronario agudo con la respuesta a la prueba de estrés (la Guía de Práctica clínica SEC SCASEST 2008 propone que en las primeras 4 a 7 semanas después del alta hospitalaria se efectúe una Ergometría o prueba de esfuerzo) $)^{3,4}$, la cuantificación de la función ventricular, la presencia de arritmias y el grado de cardiopatía isquémica. Tenemos así 3 tipos de pacientes (ver tabla I): 
Tabla I. Estratificación en función del riesgo coronario

\begin{tabular}{|c|c|c|c|c|c|}
\hline & $\begin{array}{c}\text { Prueba de } \\
\text { esfuerzo }\end{array}$ & $\begin{array}{l}\text { Capacidad } \\
\text { funcional }\end{array}$ & $\begin{array}{l}\text { Fracción de } \\
\text { eyección }\end{array}$ & Arritmias severas & $\begin{array}{c}\text { Enfermedad } \\
\text { coronaria severa } \\
\text { no revascularizable }\end{array}$ \\
\hline Riesgo bajo & $\begin{array}{l}\text { Clínica y } \\
\text { eléctricamente } \\
\text { negativa }\end{array}$ & $>7$ METS & $\geq 50 \%$ & Ausencia & No \\
\hline Riesgo medio & $\begin{array}{l}\text { Clínica y/o } \\
\text { eléctricamente } \\
\text { positiva a partir } \\
\text { del } 5 .^{\circ} \text { minuto }\end{array}$ & $\begin{array}{l}\text { Entre } 5 \text { y } 6,9 \\
\text { METS }\end{array}$ & Entre $36 \%-49 \%$ & Ausencia & No \\
\hline $\begin{array}{l}\text { Riesgo alto } \\
\text { (si presenta } \\
\text { cualquiera de } \\
\text { los siguientes) }\end{array}$ & $\begin{array}{l}\text { Clínica y } \\
\text { eléctricamente } \\
\text { positiva precoz, } \\
\text { o respuesta } \\
\text { hipotensiva }\end{array}$ & $<5$ METS & $<35 \%$ & Presencia & Sí \\
\hline
\end{tabular}

Teniendo en cuenta esta clasificación de riesgo, Sosa Rodríguez propone las siguientes recomendaciones ${ }^{5}$ :

- Los pacientes de bajo riesgo: en general pueden desarrollar la mayoría de las actividades laborales, sin superar inicialmente el $50 \%$ de la capacidad máxima de ejercicio, y puede aumentar de forma progresiva hasta el $80 \%$ de esta, si no aparecen complicaciones.

- Los pacientes de riesgo intermedio: pueden iniciar actividades laborales que no superen el $50 \%$ de la capacidad máxima de ejercicio, incluyéndose trabajos manuales estáticos.

- Los pacientes de alto riesgo: podrían realizar trabajos sedentarios si su capacidad de ejercicio superase los 4 METS sin síntomas.

Podríamos plantearnos el uso del pulsómetro en aquellos casos de riesgo bajo, como una herramienta que ayudase al paciente a convencerse de su capacidad laboral. Nunca como una herramienta orientada a forzar un alta médica.

Además el riesgo de estos pacientes debe evaluarse periódicamente, anualmente los pacientes de bajo riesgo y semestralmente en los pacientes de riesgo mayor.

\section{¿EN QUÉ TIPO DE PROFESIONES?}

De forma general podríamos plantearnos el uso del pulsómetro en aquellos casos.

- Con requerimientos energéticos compatibles con las limitaciones del paciente. Utilizaremos las distintas tablas (Astrand ${ }^{6,7}$, NTP $323^{8}$, etc.) que relacionan la capacidad funcional del paciente (que nos vendrá expresada en METs en la ergometría convencional o en $\mathrm{ml} / \mathrm{kg} / \mathrm{min}$ en la ergometría con determinación del consumo de $\mathrm{O}_{2}$ ) con el metabolismo requerido para distintas actividades ocupacionales y recreativas. En general, si la actividad laboral va a tener una duración en torno a ocho horas diarias, los gastos energéticos de esta no deberían sobrepasar el 50\% de la capacidad calculada en la ergometría prueba de esfuerzo.

- En profesiones en las que el ejercicio físico fuese básicamente aeróbico. Los ejercicios anaeróbicos producen una elevación de la presión arterial. En un paciente cardiópata este aumento de la postcarga ventricular puede ser mal tolerado y su uso debe ser restringido y realizado bajo control. En el ejercicio aeróbico (como caminar, correr, nadar, remar o montar en bicicleta) la presión 
arterial sistólica aumenta ligeramente, pero las presiones arteriales diastólica y media no se modifican de forma importante. ${ }^{9}$

- En profesiones no sometidas a estrés emocional intenso.

- En jornadas laborales que no sobrepasen las 8 horas o que impliquen cambios de turno (especialmente nocturnos).

- En trabajos que no se realicen al aire libre en condiciones de frío (ha de evitarse en lo posible el viento frío de cara), calor o humedad extremos.

- En trabajos donde los requerimientos físicos no dependan principalmente de los MMSS (se toleran peor).

- En trabajos en los que se realicen esfuerzos físicos postprandiales.

\section{¿CUÁLES SERÍAN LOS MÁRGENES DE SEGURIDAD?}

La intensidad a la que se realiza el ejercicio físico podemos controlarla por métodos subjetivos u objetivos.

Entre los primeros está la percepción subjetiva de esfuerzo del paciente. Se ha comprobado que existe una relación entre dicha percepción y el grado real de esfuerzo en términos de aumento de la frecuencia cardíaca, ventilación pulmonar y producción de lactatos. La escala de Borg, muy utilizada en rehabilitación cardiaca y en medicina del deporte, gradúa dicha percepción del esfuerzo ${ }^{10}$ en niveles que van desde el extraordinariamente ligero al extraordinariamente duro.

Entre los segundos los dos principales indicadores de la intensidad del ejercicio son la frecuencia cardiaca (FC) y el consumo de oxígeno $\left(\mathrm{VO}_{2}\right)$. Pero se diferencian en un aspecto muy relevante a la hora de su control para el entrenamiento; la FC se puede controlar mediante un pulsómetro (ha demostrado más fiabilidad que la toma manual del pulso ${ }^{11}$ ), mientras que el $\mathrm{VO}_{2}$ no se puede controlar de manera continua $\mathrm{y}$ de forma tan simple al requerir mediciones en laboratorio. Se ha visto que existe una correlación entre la FC y el $\mathrm{VO}_{2}$. Más en concreto, entre la FCR (Frecuencia cardiaca de reserva $=$ Frecuencia cardiaca máxima - frecuencia cardiaca de reposo) y el $\mathrm{VO}_{2} \mathrm{R}$ (volumen de reserva consumido de oxígeno $=\mathrm{VO}_{2}$ máx y $\mathrm{VO}_{2}$ reposo) con una proporción 1:1 independientemente de la intensidad de trabajo ${ }^{12}$. De tal forma que para estimar la intensidad del entrenamiento se pueden utilizar ambos parámetros indistintamente.

Para conocer los rangos de FC recomendables utilizamos los resultados de las pruebas de esfuerzo. En pacientes cardíacos no debe utilizarse fórmulas que estimen la frecuencia máxima teórica. No debe utilizarse ni las fórmulas clásicas como la de FC máx = 220 menos la edad en años, ni las más modernas como las de Tanaka ${ }^{13}$ (FC máx: $208-(0,7 \mathrm{x}$ edad $)$ o $\operatorname{Inbar}^{14}(\mathrm{FC} \max =205.8-0.685(\mathrm{edad}))$. Esto se debe a que es conocido que la edad es el principal factor en modular la FC $\max ^{5-18}$, pero también influyen otros factores como la obesidad ${ }^{19}$, nivel de entrenamiento ${ }^{20-21}$, sexo ${ }^{20}$ o el tipo de ejercicio ${ }^{21}$, entre otros. Mediante la prueba de esfuerzo, el especialista detecta la situación concreta del corazón y el estado de forma del paciente. En general, se recomienda $^{22}$ no sobrepasar inicialmente el $75 \%$ de la frecuencia cardiaca máxima alcanzada en caso de no presentarse ninguna alteración en la ergometría (ej: no sobrepasar $105 \mathrm{lpm}$ si se alcanzan $140 \mathrm{lpm}$ sin angina) o el 75\% de la frecuencia a la que se presenta la angina (ej. no sobrepasar $75 \mathrm{lpm}$ si la angina se presenta a 100 latidos). Con el tiempo se puede permitir llegar hasta el $80-85 \%$, pero siempre bajo la recomendación individual del especialista.

El pulsómetro, al igual que la escala de Borg, serán instrumentos de seguridad para el paciente, que le ayudarían a incorporarse antes a su vida diaria, incluyendo la vida laboral. 


\section{CONCLUSIONES}

El pulsómetro podría ser una herramienta para el paciente. Una herramienta que le convenciera de la capacidad laboral de la que el médico ya está convencido. En las altas laborales es necesario que el médico este convencido de la capacidad del paciente para la reincorporación, y en estas patologías también es importante que el paciente esté seguro de dicha capacidad.

Es posible que en el futuro las nuevas tecnologías que surgen con los avances en medicina deportiva puedan ser una herramienta para el médico inspector. Las «camisetas con Holter", utilizadas ya en medicina deportiva, y que permite prolongar el periodo de monitorización del Holter convencional de $24 \mathrm{~h}$ hasta $72 \mathrm{~h}$ o incluso 1 semana, podrían utilizarse en la valoración de la discapacidad laboral

Son necesario realizar estudios de cohortes para confirmar que la utilidad de estas herramientas en nuestro ámbito.

\section{REFERENCIAS BIBLIOGRÁFICAS}

1. Boraita A et al. Guías de práctica clínica de la Sociedad Española de Cardiología sobre la actividad física en el cardiópata. Rev Esp Cardiol 2000; 53: 684-726.

2. Instituto Nacional de la Seguridad Social: Guía de Valoración Profesional. Madrid: Instituto Nacional de la Seguridad Social 2009; 523.

3. Boudrez $\mathrm{H}$, de Backer G. Recent findings on return to work after an acute myocardial infarction or coronary artery bypass grafting. Acta Cardiol 2000; 55: 341-9.

4. Turkulin K, Cerovec D, Baborski F. Predictive markersof occupational activity in 415 post myocardial infarction patients after one-year follow-up. Eur HeartJ 1988; 9 Suppl L:103-8.

5. Sosa Rodríguez V. Estudio de la Incapacidad Laboral por Enfermedades Cardiocirculatorias. $2 .^{\circ}$ Edición. Madrid: Instituto Nacional de Medicina y Seguridad del Trabajo 1998.

6. Astrand Y. Aerobic work capacity in men and women with especial reference to age. Acta Physiol Scand 1960; 49(169 suppl): 45-60.

7. Astrand PO, Rodahl K, Texbook of work Physiology. New York: Mc Graw Hill; 1986; 391-411.

8. NTP 323: Determinación del metabolismo energético. Instituto Nacional de la Seguridad e Higiene en el Trabajo. http://www.insht.es/InshtWeb/Contenidos/Documentacion/Fichas Técnicas/NTP/ Ficheros/301. a400/ntp-323.pdf.

9. Helfant RH, DeVilla MA, Meister SG. Effect of sustained isometric handgrip exercise on left ventricular performance. Circulation 1971; 44: 982-993.

10. Borg GAV. Biological bases of perceived exection. Med Sai Sport Exer 1982; 14: 377-381.

11. Karvonen M, Vuorima T. Heart rate and exercise intensity during sports activities: Practical application. Sports Med. 1988;5:303-12.

12. Abellán J et al. Guía para la prescripción de ejercicio físico en pacientes con riesgo cardiovascular. Sociedad Española de Hipertensión y Liga Española para la Lucha contra la Hipertensión Arterial. 2010. Pg 20.

13. Tanaka, H., Monahan, K.G. and Seals, D.S. Age - predicted maximal heart rate revisited. J Am Coll Cardiol 2001;37:153-6.

14. Inbar, O. Oten, A., Scheinowitz, M., Rotstein, A., Dlin, R. and Casaburi, R. Normal cardiopulmonary responses during incremental exercise in 20-70-yr-old men. Med Sci Sport Exerc 1994;26(5):538-546.

15. Gellish RL, Goslin BR, Olson RE, McDonald A, Russi GD, Moudgil V.K. Longitudinal modeling of the relationship between age and maximal heart rate. Med Sci Sports Exerc. 2007; 39:822-9.

16. Silva VA, Bottaro M, Justino MA, Ribeiro MM, Lima RM, Oliveira R.J. Maximum heart rate in Brazilian elderly women: Comparing measured and predicted values. Arq Bras Cardiol. 2007; 88:314-20.

17. Balmer J, Potter CR, Bird SR, Davison R.C. Age-related changes in maximal power and maximal heart rate recorded during a ramped test in 114 cyclists age 15-73 years. J Aging Phys Act. 2005; 13:75-86.

18. Balmer J, Bird S, Davison R. Indoor 16.1-km time-trial performance in cyclists aged 25-63 years. J Sports Sci. $2008 ; 26: 57-62$.

19. Fornitano LD, Godoy M.F. Exercise testing in individuals with morbid obesity. Obes Surg. 2010; 20:583-8. 
20. Whyte GP, George K, Shave R, Middleton N, Nevill A.M. Training induced changes in maximum heart rate. Int J Sports Med. 2008; 29:129-33.

21. Zavorsky G.S. Evidence and possible mechanisms of altered maximum heart rate with endurance training and tapering. Sports Med. 2000; 29:13-26.

22. Dejong AT, Bonzheim K, Franklin BA, Saltarelli W. Cardiorespiratory responses to maximal arm and leg exercise in national-class marathon runners. Phys Sportsmed. 2009; 37:120-6.

23. http://www.fundaciondelcorazon.com/informacion-para-pacientes/prevencion-secundaria/cardiopatiaisquemica/ejercicio-fisico.html.

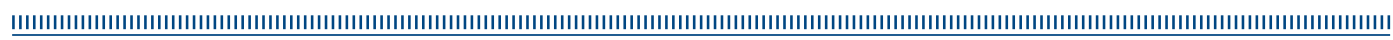

